\title{
Endocarditis and monocular blindness
}

\author{
Jack Andrews, ${ }^{1,2}$ Shyamanga Borooah ${ }^{3}$
}

${ }^{1}$ Department of Cardiovascular Sciences, University of Edinburgh, Edinburgh, Lothian, UK

${ }^{2}$ Royal Infirmary of Edinburgh, Edinburgh Heart Centre, Edinburgh, Lothian, UK

${ }^{3}$ Department of Opthalmology, Princess Alexandra Eye Pavilion, Edinburgh, Edinburgh, UK

Correspondence to Dr Jack Andrews, jackandrews@nhs.net

Accepted 7 October 2016

\section{SUMMARY}

A man aged 77 years with postrenal transplant lymphoproliferative disease was admitted with high fever, elevated inflammatory markers and a heart murmur. Blood cultures grew Enterococcus faecalis and he was found to have mitral valve endocarditis on echocardiogram and subsequently started on appropriate antibiotics. 5 days into treatment, he developed ocular symptoms and 3 days later, he had irreversible monocular visual loss. He was seen by the ophthalmology team who diagnosed endogenous endopthalmitis secondary to bacteraemic spread from his endocarditis. Despite treatment with intravitreal antibiotics and prolonged systemic antibiotics, his sight did not recover. Although septic emboli are common in endocarditis, endogenous endophthalmitis is rarely reported and frequently results in visual loss. Early treatment confers an improved prognosis.

\section{BACKGROUND}

Endogenous endophthalmitis is a rare but serious complication of endocarditis. It has been reported that up to half of all cases are misdiagnosed at presentation and few recover useful vision. ${ }^{1}$ Early recognition of new visual impairment in patients with endogenous endophthalmitis may prevent permanent visual loss through prompt diagnosis and treatment.

\section{CASE PRESENTATION}

A man aged 77 years with postrenal transplant lymphoproliferative disease was admitted with a high fever. He had recently finished a course of 'R-CHOP' chemotherapy.

Clinical examination revealed a resting tachycardia and a pansystolic murmur radiating to the axilla. Investigations demonstrated an elevated $\mathrm{C}$ reactive protein (CRP). Blood cultures grew Enteroccous faecalis. Chest radiograph and ECG were unremarkable. Transthoracic and subsequent transoesophageal echocardiography confirmed a small $(<1 \mathrm{~cm})$ vegetation on the posterior mitral valve leaflet (figure 1). He was started on intravenous amoxicillin as per microbiological advice and made prompt clinical improvement.

Five days into treatment, he reported blurring of vision in his right eye only. Ocular examination found mild scleral injection only and he was prescribed lubricating eye drops. After 3 days, his vision had deteriorated further, he had a mild discomfort in the eye and a slowed pupillary response. Examination found a best-corrected visual acuity of $6 / 18$ in the right and 6/6 in the left eye. He was urgently referred to ophthalmology who took the retinal picture (figure 2) and angiogram (figure 3 ).

\section{INVESTIGATIONS}

The patient had an elevated CRP and white cell count. ECG and chest X-ray were unremarkable. Blood cultures grew Enterococcus faecalis. Transthoracic and transoesophageal echocardiogram confirmed a small vegetation on the posterior mitral valve leaflet. A CT scan brain was considered to exclude cerebral emboli but ultimately was not requested as the visual loss was monocular and the patient had localised eye discomfort.

\section{DIFFERENTIAL DIAGNOSIS}

Based on the clinical and investigative picture, the possible diagnoses were widespread and included; endogenous endophthalmitis, vasculitic uveitis, cytomegalovirus uveitis, syphilitic uveitis, intraocular malignancy and cerebral emboli.

\section{TREATMENT}

The patient's antibiotic treatment was complex. $\mathrm{He}$ received systemic intravenous amoxicillin (6-week total duration) on the basis that he had a penicillinsensitive enterococcus bacteraemia. Synergistic gentamicin was avoided due to deteriorating renal function. This regime was decided after careful discussion with the microbiology and renal teams. Ophthalmic and microbiological teams employed an ocular antibiotic regime of oral ciprofloxacin and intravitreal vancomycin and ceftriaxone. Empirical treatment was employed while awaiting intravitreal culture results.

On a general level, intravitreal antibiotic administration may need to be repeated and occasionally, vitrectomy is required in severe non-responsive and progressive cases. Once the antibiotic therapy is completed, topical and systemic corticosteroids may be used to treat the infection.

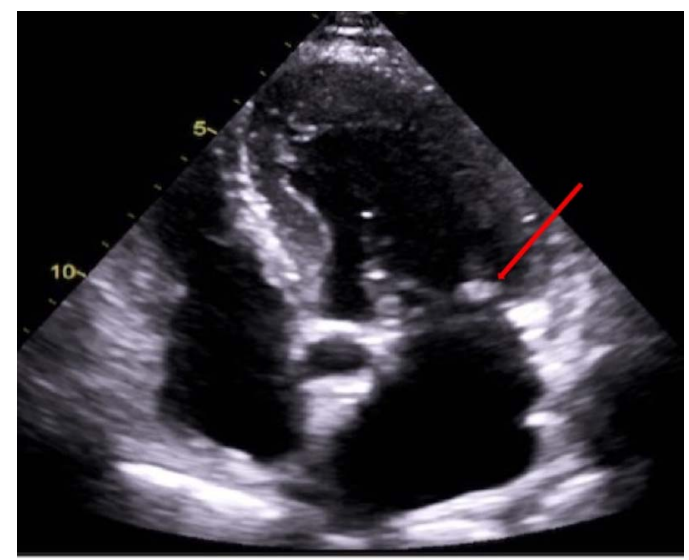

Figure 1 Transthoracic echocardiographic image of mitral valve vegetation (red arrow) on posterior mitral valve leaflet in apical five-chamber view. 


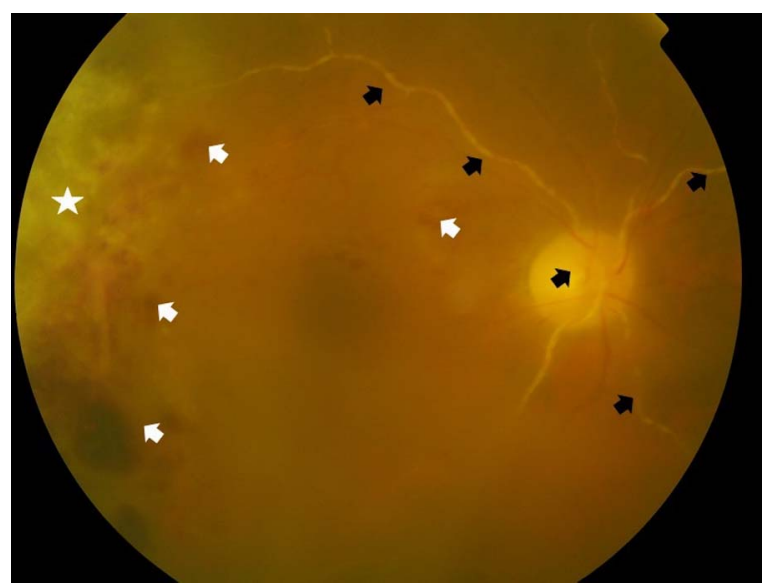

Figure 2 A normal optic disc with a markedly attenuated arterial circulation resulting in vascular sheathing (white arrows). The macula demonstrates scattered Roth spots (black arrows) and ischaemic retinitis (star) in the temporal watershed area. The retinal findings result from the combination of ischaemic retinal arterial occlusion and an immunogenic response from septic emboli.

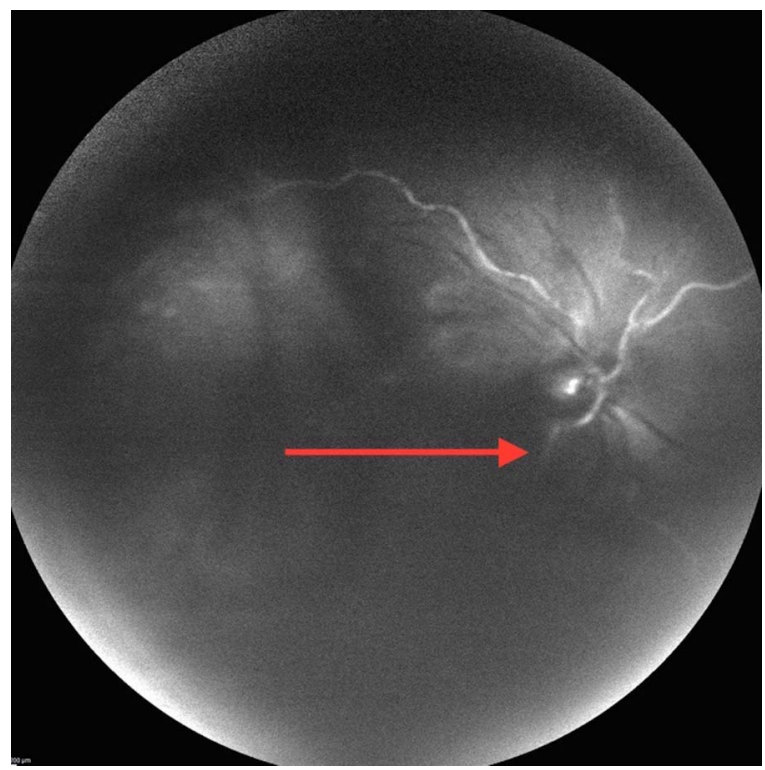

Figure 3 Retinal angiogram showing retinal vascular occlusion (red arrow).

\section{OUTCOME AND FOLLOW-UP}

The patient remained with loss of vision in the affected eye but made a good recovery from his endocarditis with successful sterilisation after prolonged antibiotic treatment. Regrettably, the patient has recently passed away from an aggressive form of lymphoma as a result of post-transplant lymphoproliferative disease.

\section{DISCUSSION}

The incidence of infective endocarditis is increasing and endogenous endophthalmitis from septic emboli is a serious but rarely reported complication. A retrospective 10-year review identified only 28 cases with $40 \%$ of these attributable to bacteraemia from endocarditis. ${ }^{2}$ In those with Staphylococcus aureus bacteraemia and ocular involvement, infective endocarditis was found to be an independent risk factor for the development of endopthalmitis. ${ }^{3}$ The streptococcus species (as seen in this case) are the most commonly implicated bacterial pathogens, reported to cause between $30 \%$ and $50 \%$ of cases. ${ }^{1}$

Our patient's intravitreal culture was negative, but this was not surprising, given that tissue sampling was performed after the administration of high-dose intravenous amoxicillin. The decision to omit synergistic gentamicin (as per the European Society of Cardiology guidelines) was made due to the deteriorating function of the patient's renal transplant and after discussion with renal and microbiology teams.

\section{Learning points}

- Endogenous endophthalmitis is a rarely reported but serious complication of endocarditis.

- Peripheral embolic phenomena in the context of endocarditis may indicate uncontrolled infection and confers a worse prognosis.

- Adherence to international guidelines for the management of endocarditis is advised.

- Urgent ophthalmic referral in all patients who develop ocular symptoms during bacteraemia is strongly recommended. Failure to do so may result in irreversible visual loss.

Acknowledgements Medical photography department, Princess Alexandra Eye Pavilion, Edinburgh, UK.

Contributors JA planned, conducted and reported the case with help from SB.

Competing interests None declared.

Patient consent Obtained.

Provenance and peer review Not commissioned; externally peer reviewed.

\section{REFERENCES}

1 Binder MI, Chua J, Kaiser PA, et al. Endogenous endophthalmitis: an 18-year review of culture-positive cases at a tertiary care center. Medicine (Baltimore) 2003;82: 97-105.

2 Okada AA, Johnson RP, Liles WC, et al. Endogenous bacterial endophthalmitis. Report of a ten-year retrospective study. Ophthalmology 1994;101:832.

3 Jung J, Lee J, Yu SN, et al. Incidence and risk factors of ocular infection caused by Staphylococcus aureus bacteremia. Antimicrob Agents Chemother 2016;60: 2012-17. 
Copyright 2016 BMJ Publishing Group. All rights reserved. For permission to reuse any of this content visit http://group.bmj.com/group/rights-licensing/permissions.

BMJ Case Report Fellows may re-use this article for personal use and teaching without any further permission.

Become a Fellow of BMJ Case Reports today and you can:

- Submit as many cases as you like

- Enjoy fast sympathetic peer review and rapid publication of accepted articles

- Access all the published articles

- Re-use any of the published material for personal use and teaching without further permission

For information on Institutional Fellowships contact consortiasales@bmjgroup.com

Visit casereports.bmj.com for more articles like this and to become a Fellow 